\title{
Coliftings and Gorenstein injective modules
}

\author{
By
}

\author{
Edgar E. ENOCHS and Overtoun M. JENDA
}

\section{Introduction}

Throughout this paper, $R$ will denote a commutative noetherian ring.

We recall that an $R$-module $M$ is said to be Gorenstein injective if and only if for any $R$-module $Q$ of finite injective or projective dimension, $\operatorname{Ext}_{R}^{i}(Q, M), \operatorname{Ext}_{i}^{R}(Q, M)$ for all $i \geq 1$ and $\operatorname{Ext}_{R}^{0}(Q, M), \operatorname{Ext}_{0}^{R}(Q, M)$ vanish (see Enochs-Jenda [4] for equivalent definitions). We note that Gorenstein injective modules are dual to Auslander's maximal Cohen-Macaulay modules. While the latter modules are studied in the category of finitely generated modules, Gorenstein injective modules are rarely finitely generated (see [4]).

The aim of this paper is to study Maranda type of results for Gorenstein injective modules. We note that these type of results have been generalized to maximal Cohen-Macaulay modules over $R$-algebras where $R$ is a complete local Gorenstein ring (see Auslander-Ding-Soldberg [1] and Ding-Soldberg [2]).

We recall that an $R$-module $M$ is said to be strongly indecomposable if $\operatorname{End}(M)$ is a local ring, and $M$ is said to be reduced if it has no nonzero injective submodules. We note that every strongly indecomposable module is indecomposable and thus reduced.

If $x$ is an $R$-regular element that is not regular on an $R$-module $M$, then $r_{x}(M)$ will denote the least integer $r$ such that $x^{r} \cdot \operatorname{Ext}^{1}(, M)=0$. We will show that if $r_{x}(M)$ is finite, $r \geq r_{x}(M) \geq 0$ and $M$ is a strongly indecomposable Gorenstein injective $R$-module such that $\operatorname{Hom}_{R}\left(\frac{R}{x^{r} R}, M\right) \cong \operatorname{Hom}_{R}\left(\frac{R}{x^{r} R}, N\right)$ for some strongly indecomposable Gorenstein injective $R$-module $N$ with $r \geq r_{x}(N) \geq 0$, then $M \cong N$ or $N \cong S(M) \cong S^{2}(N)$ (showing that $N$ has periodic injective resolution of period 2) where $S^{i}(M)$ denotes the $i^{\text {th }}$ cosyzygy of $M$. Furthermore, if $r_{x}(M)$ is finite and $r \geq r_{x}(M) \geq 0$ and $M$ is strongly indecomposable, then $\operatorname{Hom}_{R}\left(\frac{R}{x^{r} \bar{R}}, M\right)$ is indecomposable or $\operatorname{Hom}_{R}\left(\frac{R}{x^{r} \bar{R}}, M\right)$ $\cong L \oplus S_{R / x^{r} R}(L)$ for some indecomposable $L$ (Theorem 3.3). This result is obtained without requiring the Gorenstein condition on the ring. As an easy consequence, we get that if $x$ is $R$-regular, and $E, E^{\prime}$ are indecomposable injective $R$-modules with $\operatorname{Hom}_{R}\left(\frac{R}{x R}, E\right) \cong \operatorname{Hom}_{R}\left(\frac{R}{x R}, E^{\prime}\right) \neq 0$, then $E \cong E^{\prime}$.

A linear map $\psi: M \rightarrow G$ where $G$ is a Gorenstein injective $R$-module is said to be a Gorenstein injective preenvelope if $\operatorname{Hom}\left(G, G^{\prime}\right) \rightarrow \operatorname{Hom}\left(M, G^{\prime}\right) \rightarrow 0$ is exact for all 
Gorenstein injective $R$-modules $G^{\prime}$. If furthermore, $f \circ \psi=\psi$ for $f \in \operatorname{Hom}(G, G)$ implies $f$ is an automorphism, then $\psi$ is called a Gorenstein injective envelope. It was shown in Enochs-Jenda-Xu [8] that if $R$ is $n$-Gorenstein (that is, the injective dimension of $R$ over itself is at most $n$ ), then every $R$-module has a Gorenstein injective envelope. Gorenstein injective envelopes are unique up to isomorphism. Furthermore, since every injective module is Gorenstein injective, we see that Gorenstein injective envelopes are monomorphisms. So if $\psi: M \rightarrow G$ is a Gorenstein injective envelope, we say that $G$, denoted $G(M)$, is the Gorenstein injective envelope of $M$. Likewise, $E(M)$ will denote the injective envelope of $M$.

A linear map $\psi: E \rightarrow M$ where $E$ is an injective $R$-module is said to be an injective precover if $\operatorname{Hom}\left(E^{\prime}, E\right) \rightarrow \operatorname{Hom}\left(E^{\prime}, M\right) \rightarrow 0$ is exact for all injective $R$-modules $E^{\prime}$. If furthermore, $\psi \circ f=\psi$ for $f \in \operatorname{Hom}(E, E)$ implies $f$ is an automorphism, then $\psi$ is called an injective cover. Injective covers were shown to exist over noetherian rings in Enochs [3]. Again, injective covers are unique but they are not surjective in general.

If $R \rightarrow S$ is a ring homomorphism and $L$ is an $S$-module, then an $R$-module $M$ is said to be a colifting of $L$ to $R$ if
1) $L \cong \operatorname{Hom}_{R}(S, M)$
2) $\operatorname{Ext}_{R}^{i}(S, M)=0$ for all $i \geq 1$.

If $L$ is a direct summand of $\operatorname{Hom}_{R}(S, M)$ satisfying (2), then $M$ is said to be a weak colifting of $L$. We say that $L$ is coliftable or weakly coliftable to $R$, respectively, if there is such an $R$-module $M$. Clearly, every coliftable $S$-module is weakly coliftable. But weakly coliftable modules need not be coliftable (see Example 4.3). We note that if $M$ is Gorenstein injective, then $\operatorname{Ext}_{R}^{i}(S, M)=0$ for all $i \geq 1$ whenever $p d_{R} S<\infty$. Thus if $M$ is Gorenstein injective and $p d_{R} S<\infty$, then $M$ is a colifting of an $S$-module $L$ if and only if $L \cong \operatorname{Hom}_{R}(S, M)$.

If $x$ is $R$-regular, then we will denote $\frac{R}{x R}$ by $\bar{R}$. The aim of Section 4 is to study coliftings of $\bar{R}$-modules. We show that if $M$ is a strongly indecomposable Gorenstein injective module over an $n$-Gorenstein ring $R$ and $M$ is a colifting of a nonzero $\bar{R}$-module $L$ such that $x \cdot \operatorname{Ext}^{1}(, M)=0$, then $S_{R}\left(\operatorname{Hom}_{R}\left(\bar{R}, S^{-1}(M)\right)\right)$ is a reduced Gorenstein injective $R$-module and $G_{R}(L) \cong M \oplus S^{-1}(M)$ where $G_{R}(L)$ and $S^{-1}(M)$ denote the Gorenstein injective envelope of the $R$-module $L$ and the kernel of the injective cover of $M$, respectively (Theorem 4.2).

An $R$-module $M$ is said to be an essential colifting of an $\bar{R}$-module $L$ if $M$ is a colifting of $L$ and the $R$-imbedding $L \subseteq M$ is an essential extension. We also say that $L$ is essentially coliftable. We argue that if $R$ is $n$-Gorenstein and $M$ is an essential colifting of an $\bar{R}$-module to $R$, then $\operatorname{Hom}_{R}\left(\bar{R}, G_{R}(M)\right) \cong G_{\bar{R}}(\operatorname{Hom}(\bar{R}, M))$ (Proposition 4.6). We use this Lemma to obtain an analog of Proposition 5.2 of Auslander-Ding-Soldberg [1], namely that if $L$ is essentially coliftable to $R$, then $G_{\bar{R}}(L)$ and $\frac{G_{R}(L)}{L}$ are also coliftable to $R$ (Theorem 4.7). We then characterize essentially coliftable $\bar{R}$-modules over 2 -Gorenstein rings in Theorem 4.9 giving us an analog of Proposition 4.3 of [1]. Finally, we characterize weakly coliftable 
$\bar{R}$-modules in Theorem 4.11 .

\section{Preliminaries}

We start with the following easy

Lemma 2.1. Let $\sigma: M \rightarrow N$ be a surjective homomorphism of $R$-modules $M$ and $N$. If $\sigma$ factors through $E(M)$, then $N$ is a direct summand of $\frac{E(M)}{\operatorname{Ker} \sigma}$.

Proof. Since $\sigma$ factors through $E(M)$, we have the following commutative diagram

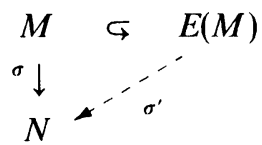

with $\sigma^{\prime}(\operatorname{Ker} \sigma)=0$. So we have the followed induced commutative diagram

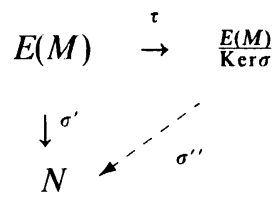

But then we have the following commutative diagram

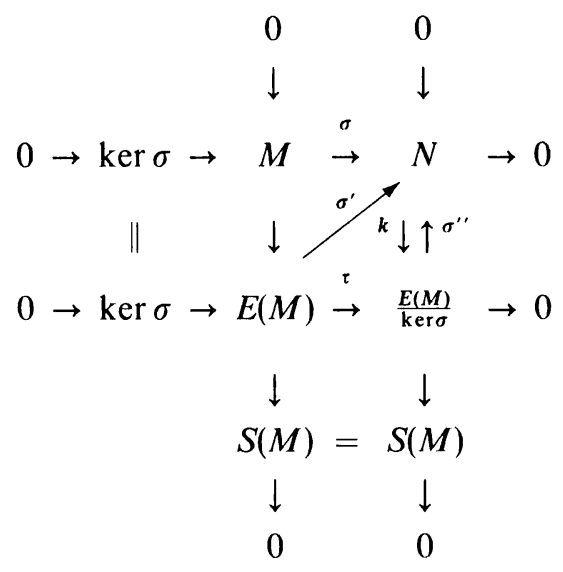

where $\sigma^{\prime \prime} \circ k \circ \sigma=\sigma^{\prime \prime} \circ \tau_{\mid M}=\sigma_{\mid M}^{\prime}=\sigma$. But $\sigma$ is onto. So $\sigma^{\prime \prime} \circ k=i d_{N}$. Thus the result follows.

Proposition 2.2 (Hilton [9]). Let $\sigma: M \rightarrow N$ be a homomorphism of $R$ modules. Then the following are equivalent.

1) $\sigma$ factors through an injective $R$-module. 
2) $\sigma$ factors through the injective envelope of $M$.

3) $\sigma$ factors through the injective cover of $N$.

4) $\sigma \cdot \operatorname{Ext}^{1}(, M)=0$

5) $\sigma \cdot(0 \rightarrow M \rightarrow E(M) \rightarrow S(M) \rightarrow 0)=0$ in $\operatorname{Ext}^{1}(S(M), N)$.

Furthermore, if $\sigma$ is onto and factors through an injective $R$-module, then $\frac{E(M)}{\operatorname{Ker} \sigma} \cong S(M) \oplus N$.

Proof. $1 \Rightarrow 2$. Let $\sigma$ factor through an injective $R$-module $E$. Then the diagram

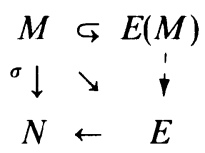

can be completed to a commutative diagram since $E$ is injective. So (2) follows.

$2 \Rightarrow 3$. Let $E \rightarrow N$ be the injective cover of $N$. Then the diagram

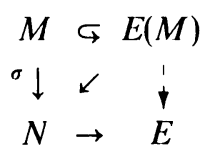

can be completed to a commutative diagram. So (3) follows.

$3 \Rightarrow 1$ is trivial.

$1 \Rightarrow 4$. Let $\sigma: M \stackrel{\psi}{\rightarrow} \underset{E^{\tau}}{\rightarrow} N$ be the factorization of $\sigma$ through an injective $R$-module $E$. Then we have the following induced commutative diagram

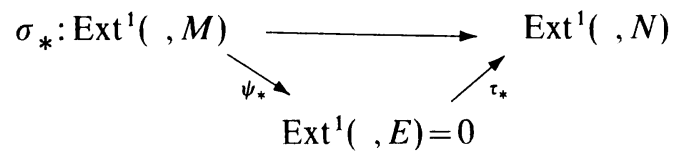

Thus $\sigma_{*}=\tau_{*} \circ \psi_{*}=0$ and so $\sigma \cdot \operatorname{Ext}^{1}(M)=0$.

$4 \Rightarrow 5$. We simply note that $0 \rightarrow M \rightarrow E(M) \rightarrow S(M) \rightarrow 0 \in \operatorname{Ext}^{1}(S(M), M)$ and so $\sigma \cdot(0 \rightarrow M \rightarrow E(M) \rightarrow S(M) \rightarrow 0)=0$ in $\operatorname{Ext}^{1}(S(M), N)$ since $\sigma \cdot \operatorname{Ext}^{1}(, M)=0$.

$5 \Rightarrow 2$. We again consider the exact sequenct $0 \rightarrow M \rightarrow E(M) \rightarrow S(M) \rightarrow 0$. We have the long exact sequence

$$
0 \rightarrow \operatorname{Hom}(S(M), N) \rightarrow \operatorname{Hom}(E(M), N) \rightarrow \operatorname{Hom}(M, N) \rightarrow \operatorname{Ext}^{1}(S(M), N) \rightarrow \cdots
$$

But $\sigma \cdot(0 \rightarrow M \rightarrow E(M) \rightarrow S(M) \rightarrow 0)=0$ in $\operatorname{Ext}^{1}(S(M), N)$. So the diagram 


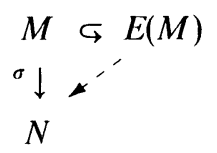

can be completed to a commutative diagram and thus (2) follows.

Now suppose $\sigma: M \rightarrow N$ is onto and factors through an injective $R$-module. Then $\sigma$ factors through $E(M)$ by the above. So $\frac{E(M)}{\operatorname{Ker} \sigma} \cong S(M) \oplus N$ by Lemma 2.1 above.

Corollary 2.3. Let $x$ be $R$-regular and $M$ be an $x$-divisible $R$-module. If $x \cdot \operatorname{Ext}^{1}(, M)=0$, then $\frac{E(M)}{\operatorname{Hom}_{R}(R, M)} \cong S(M) \oplus M$.

Proof. Let multiplication by $x$ on $M$ be the map $\sigma$ in the Proposition above. Then $\sigma$ is onto since $M$ is $x$-divisible. So the result follows immediately from the Proposition.

Corollary 2.4. Let $x$ be $R$-regular and $M$ be an $x$-divisible $R$-module such that $\operatorname{Hom}_{R}(\bar{R}, M) \subseteq M$ is an essential extension. If $x \cdot \operatorname{Ext}^{1}(, M)=0$, then $S\left(\operatorname{Hom}_{R}(\bar{R}, M)\right)$ $\cong S(M) \oplus M$.

Proof. We simply note that in this case $E(M) \cong E\left(\operatorname{Hom}_{R}(\bar{R}, M)\right)$ and so the result follows from Corollary 2.3 .

\section{Gorenstein injective modules and regular elements}

If $x$ is an $R$-regular element and $M$ is a Gorenstein injective $R$-module, then $\operatorname{Ext}^{1}(\bar{R}, M)=0$ since $p d \bar{R} \leq 1$. Thus we have the exact sequence $0 \rightarrow \operatorname{Hom}_{R}(\bar{R}, M) \rightarrow M$ $\stackrel{x}{\rightarrow} M \rightarrow 0$. In particular, we have that Gorenstein injective modules are $x$-divisible.

We start with the following

Lemma 3.1. Let $x$ be $R$-regular and $M$ be a reduced Gorenstein injective $R$-module such that $\operatorname{Hom}_{R}(\bar{R}, M) \neq 0$ and $x \cdot \operatorname{Ext}^{1}(, M)=0$, then $\operatorname{Hom}_{R}(\bar{R}, M)$ is an essential $R$-submodule of $M$.

Proof. We first note that $E(M) \cong E\left(\operatorname{Hom}_{R}(\bar{R}, M)\right) \oplus E$ for some injective $R$-module $E$. And so by Corollary 2.3 , we have that $S\left(\operatorname{Hom}_{R}(\bar{R}, M)\right) \oplus E \cong S(M) \oplus M$. If $M$ is reduced, then $S(M)$ is reduced. It is then easy to see that $S(M) \oplus M$ is also reduced. Hence $E=0$ and $E(M) \cong E\left(\operatorname{Hom}_{R}(\bar{R}, M)\right)$. So the result follows.

We also need the following

Lemma 3.2. Let $x$ be $R$-regular and $L$ be an $\bar{R}$-module. Then $L$ is weakly coliftable to $R$ if and only if 


$$
\operatorname{Hom}\left(\bar{R}, S_{R}(L)\right) \cong L \oplus S_{\bar{R}}(L)
$$

Proof. Let $M$ be a weak colifting of $L$ to $R$. Then we have the following commutative diagram

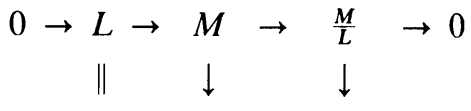

$$
\begin{aligned}
& 0 \rightarrow L \rightarrow E(L) \rightarrow S_{R}(L) \rightarrow 0
\end{aligned}
$$

We now apply $\operatorname{Hom}_{R}(\bar{R},-)$ to the diagram to get

$$
\begin{aligned}
& 0_{L}: 0 \rightarrow L \stackrel{i}{\rightarrow} \operatorname{Hom}_{R}(\bar{R}, M) \rightarrow \operatorname{Hom}_{R}\left(\bar{R}, \frac{M}{L}\right) \rightarrow L \rightarrow 0
\end{aligned}
$$

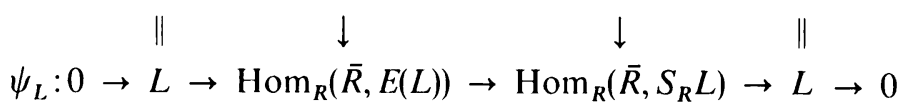

since $\operatorname{Hom}_{R}(\bar{R}, L) \cong \operatorname{Ext}_{R}^{1}(\bar{R}, L) \cong L$.

But $\operatorname{Hom}_{R}(\bar{R}, E(L))=E_{\bar{R}}(L)$. So $\left.\operatorname{Ext}_{R}^{1}\left(L, S_{\bar{R}} L\right)\right) \cong \operatorname{Ext}_{R}^{2}(L, L)$. Furthermore, we note that $\theta_{L}, \psi_{L}$ represent the same elements in $\operatorname{Ext}_{R}^{2}(L, L)$. But $i$ is a split monomorphism by assumption and so $\theta_{L}$ is zero in $\operatorname{Ext}_{\tilde{R}}^{2}(L, L)$. Therefore, $\psi_{L}$ is zero in $\operatorname{Ext}_{\bar{R}}^{1}\left(L, S_{\bar{R}}(L)\right)$. That is, $0 \rightarrow S_{\bar{R}}(L) \rightarrow \operatorname{Hom}_{R}\left(\bar{R}, S_{R}(L)\right) \rightarrow L \rightarrow 0$ is split exact and so the result follows.

The converse is trivial.

If $x$ is $R$-regular but not regular on an $R$-module $M$, then for $r \geq 0$, we set

$$
r_{x}(M)=\min \left\{r: x^{r} \cdot \operatorname{Ext}^{1}(, M)=0\right\} .
$$

If there is no such $r$, we set $r_{x}(M)=\infty$. We note that $r_{x}(M)=0$ if and only if $M$ is an injective $R$-module.

We are now in a position to state the following Maranda type of result.

Theorem 3.3. Let $x$ be $R$-regular and $M$ be an $x$-divisible $R$-module such that $r_{x}(M)$ is finite and $r \geq r_{x}(M) \geq 0$. Then

1) If $\operatorname{Hom}_{R}\left(\frac{R}{x^{r} R}, M\right) \cong \operatorname{Hom}_{R}\left(\frac{R}{x^{r} R}, N\right)$ for some $x$-divisible $R$-module $N$ with $r \geq r_{x}(N) \geq 0$ and $\operatorname{Hom}_{R}\left({ }_{x}^{R}{ }_{\bar{R}}^{R}, M\right) \subseteq M$ and $\operatorname{Hom}_{R}\left(x_{x^{r} \bar{R}}^{R}, N\right) \subseteq N$ are essential extensions, then $M \oplus S(M) \cong N \oplus S(N)$.

2) If $M$ is a strongly indecomposable Gorenstein injective $R$-module such that $\operatorname{Hom}_{R}\left(\frac{R}{x^{r} R}, M\right) \cong \operatorname{Hom}_{R}\left(\frac{R}{x^{r} R}, N\right)$ for some strongly indecomposable Gorenstein injective $R$-module $N$ with $r \geq r_{x}(N) \geq 0$, then $M \cong N$ or $N \cong S(M) \cong S^{2}(N)$.

3) If $M$ is a strongly indecomposable Gorenstein injective $R$-module, then $\operatorname{Hom}_{R}\left(\frac{R}{x^{r} \bar{R}}, M\right)$ is indecomposable or $\operatorname{Hom}_{R}\left(\frac{R}{x^{r} \bar{R}}, M\right) \cong L \oplus S_{\frac{R}{x^{r}}}(L)$ for some in- 
decomposable $\frac{R}{x^{r} R}-$ module $L$.

Proof. 1) easily follows from Corollary 2.4 .

2) If $M, N$ are strongly indecomposable, then $\operatorname{Hom}_{R}\left(\frac{R}{x^{r} R}, M\right) \subseteq M$ and $\operatorname{Hom}_{R}\left(\frac{R}{x^{r} R}, N\right) \subseteq N$ are essential extensions by Lemma 3.1 and so $M \oplus S(M) \cong N \oplus S(N)$ by part (1). But $S(M), S(N)$ are also strongly indecomposable. So the result follows by the Krull-Remak-Schmidt-Azumaya Theorem.

3) Suppose $\operatorname{Hom}_{R}\left(\frac{R}{x^{r} R}, M\right)=L \oplus L^{\prime}$. Then $S\left(\operatorname{Hom}_{R}\left(\frac{R}{x^{r} R}, M\right)\right)=S(L) \oplus S\left(L^{\prime}\right)$. But $S\left(\operatorname{Hom}_{R}\left(\frac{R}{x^{r} R}, M\right)\right) \cong S(M) \oplus M$ by Corollary 2.4 and Lemma 3.1 and $S(M)$ is also strongly indecomposable. So we may assume that $M \cong S(L)$. But $L$ is weakly coliftable and so $\operatorname{Hom}_{R}\left(\frac{R}{x^{r} R}, M\right) \cong \operatorname{Hom}_{R}\left(\frac{R}{x^{r} R}, S(L)\right) \cong L \oplus S_{\frac{R}{x^{r} R}}(L)$ by Lemma 3.2. But $M$ is a strongly indecomposable Gorenstein injective $R$-module and therefore so is $S(L)$. But then $L$ is indecomposable.

Corollary 3.4. Let $x$ be $R$-regular and $E, E^{\prime}$ be indecomposable injective $R$-modules with $\operatorname{Hom}_{R}(\bar{R}, E) \cong \operatorname{Hom}_{R}\left(\bar{R}, E^{\prime}\right) \neq 0$. Then $E \cong E^{\prime}$.

We now state the following easy properties of $r_{x}(M)$.

Proposition 3.5. 1) Let $0 \rightarrow M^{\prime \prime} \rightarrow M \rightarrow M^{\prime} \rightarrow 0$ be an exact sequence of $R$-modules such that $r_{x}\left(M^{\prime \prime}\right)$ and $r_{x}\left(M^{\prime}\right)$ are finite, then $r_{x}(M) \leq r_{x}\left(M^{\prime \prime}\right)+r_{x}\left(M^{\prime}\right)$.

2) If $M$ is an $R$-module with $r_{x}(M)<\infty$, then $r_{x}(S(M)) \leq r_{x}(M)$.

3) If $M \cong \oplus_{i=1}^{s} M_{i}$, then $r_{x}(M)=\max \left\{r_{x}\left(M_{i}\right)\right\}$.

Proof. 1) We consider the long exact sequence $\cdots \rightarrow \operatorname{Ext}^{1}\left(-, M^{\prime \prime}\right) \rightarrow \operatorname{Ext}^{1}(-$, $M) \rightarrow \operatorname{Ext}^{1}\left(-, M^{\prime}\right) \rightarrow \cdots$. If $r_{x}\left(M^{\prime \prime}\right)=m$ and $r_{x}\left(M^{\prime}\right)=n$, then $x^{m+n} \cdot \operatorname{Ext}^{1}(, M)=0$ and so $r_{x}(M) \leq m+n$.

2) If $r_{x}(M)=m$, then $x^{m} \cdot \operatorname{Ext}^{1}(, M)=0$ and so $x^{m}: M \rightarrow M$ factors through $E(M)$ by Proposition 2.2. So we have the commutative diagram

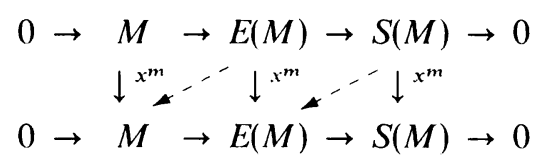

Thus $x^{m}: S(M) \rightarrow S(M) \rightarrow$ factors through $E(M)$ and hence $x^{m} \cdot \operatorname{Ext}^{1}(, S(M))=0$. That is, $r_{x}(S(M)) \leq m$.

3) This follows from the fact that $\operatorname{Ext}^{1}(, M) \cong \oplus_{i=1}^{s} \operatorname{Ext}^{1}\left(, M_{i}\right)$.

Corollary 3.6. Let $x$ be $R$-regular but not regular on a reduced Gorenstein injective $R$-module $M$. If $r_{x}(M)<\infty$, then $r_{x}(M)=r_{x}(S(M))=r_{x}\left(S^{-1}(M)\right)$. 
Proof. If $r_{x}(M)<\infty$, then $r_{x}(S(M)) \leq r_{x}(M)$ by Proposition 3.5. If $m=r_{x}(S(M))$, then $x^{m}: S(M) \rightarrow S(M)$ can be factored through the injective cover $E(M) \rightarrow S(M)$. So $x^{m}: M \rightarrow M$ can be factored through $E(M)$ as in the proof of the Proposition above. So $r_{x}(M) \leq r_{x}(S(M))$. Thus $r_{x}(M)=r_{x}(S(M))$.

Furthermore, $M=S\left(S^{-1}(M)\right)$ since $M$ is reduced and so $r_{x}(M)=r_{x}\left(S^{-1}(M)\right)$.

\section{Coliftings over $n$-Gorenstein Rings}

We start with the following result.

Lemma 4.1. Let $R$ be an n-Gorenstein ring, $x$ be $R$-regular, and $M$ be $a$ reduced Gorenstein injective $R$-module such that $x$ is not regular on $M$. Suppose $S\left(\operatorname{Hom}_{R}\left(\bar{R}, S^{-1}(M)\right)\right)$ is a reduced $R$-module. Then $S\left(\operatorname{Hom}_{R}\left(\bar{R}, S^{-1}(M)\right)\right)$ is Gorenstein injective if and only if $S\left(\operatorname{Hom}_{R}\left(\bar{R}, S^{-1}(M)\right)\right) \cong G\left(\operatorname{Hom}_{R}(\bar{R}, M)\right)$.

Proof. Since $M$ is Gorenstein injective, the injective cover $E \rightarrow M$ is surjective. So we have the exact sequence $0 \rightarrow S^{-1}(M) \rightarrow E \rightarrow M \rightarrow 0$. Thus we obtain the following commutative diagram

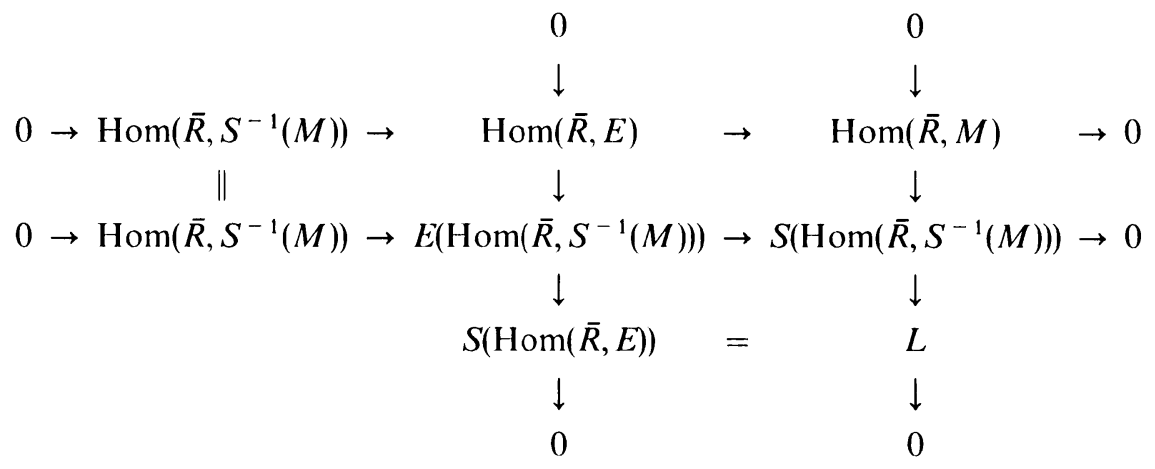

But $i d_{R} \operatorname{Hom}(\bar{R}, E) \leq 1$ since $\operatorname{Hom}(\bar{R}, E)$ is an injective $\bar{R}$-module. So $L$ is an injective $R$-module. Thus $p d L<\infty$ since $R$ is $n$-Gorenstein. Consequently, if $S\left(\operatorname{Hom}_{R}(\bar{R}\right.$, $\left.\left.S^{-1}(M)\right)\right)$ is Gorenstein injective, then $\operatorname{Hom}(\bar{R}, M) \subseteq S\left(\operatorname{Hom}_{R}\left(\bar{R}, S^{-1}(M)\right)\right)$ is a Gorenstein injective preenvelope and hence $S_{R}\left(\operatorname{Hom}_{R}\left(\bar{R}, S^{-1}(M)\right)\right) \cong G_{K}\left(\operatorname{Hom}_{R}(\bar{R}\right.$, $M)) \oplus E^{\prime}$ for some injective $R$-module $E^{\prime} . \quad$ But then $E^{\prime}=0$ since $S\left(\operatorname{Hom}_{R}\left(\bar{R}, S^{-1}(M)\right)\right)$ is reduced. The converse is trivial.

Theorem 4.2. Let $R$ be $n$-Gorenstein, $x$ be $R$-regular and $M$ be a reduced Gorenstein injective $R$-module that is a colifting of a nonzero $\bar{R}$-module $L$. If $x \cdot \operatorname{Ext}^{1}(, M)=0$, then $S_{R}\left(\operatorname{Hom}_{R}\left(\bar{R}, S^{-1}(M)\right)\right)$ is a reduced Gorenstein injective R-module and $G_{R}(L) \cong M \oplus S^{-1}(M)$. In this case, $r_{x}\left(G_{R}(L)\right)=r_{x}(M)$.

Proof. We first note that $x \cdot \operatorname{Ext}^{1}(, M)=0$ if and only if $x \cdot \operatorname{Ext}^{1}\left(, S^{-1}(M)\right)=0$ since $M \stackrel{x}{\rightarrow} M$ factors through the injective cover $E_{0} \rightarrow M \rightarrow 0$ if and only if 
$S^{-1}(M) \stackrel{x}{\rightarrow} S^{-1}(M)$ factors through the injective envelope $0 \rightarrow S^{-1}(M) \rightarrow E_{0}$. Furthemore, $S^{-1}(M)$ is also a reduced Gorenstein injective $R$-module. So $S\left(\operatorname{Hom}_{R}\left(\bar{R}, S^{-1}(M)\right)\right) \cong M \oplus S^{-1}(M)$ by Corollary 2.4 and Lemma 3.1 . Hence $S\left(\operatorname{Hom}_{R}\left(\bar{R}, S^{-1}(M)\right)\right)$ is a reduced Gorenstein injective $R$-module since $M \oplus S^{-1}(M)$ is such. So $G_{R}(L) \cong M \oplus S^{-1}(M)$ by the Lemma above.

Example 4.3. Let $R=k\left[\left[x^{2}, x^{3}\right]\right]$ with $k$ a field. Then $R$ is a Gorenstein local ring since $\{0,2,3,4, \cdots\} \subset N$ is symmetric (see Kunz [11]). In fact, $R$ is a 1 -Gorenstein domain.

Now let $G=k+k x^{-1}+k x^{-2}+\cdots$. Then $G$ is a divisible $R$-module and thus it is Gorenstein injective since $R$ is 1 -Gorenstein. Moreover, $\operatorname{Hom}_{R}(G, G) \cong k[[x]]$. Hence $G$ is a strongly indecomposable Gorenstein injective $R$-module. Furthermore, $\operatorname{Hom}_{R}\left(\frac{R}{x^{2} R}, G\right)=k+k x^{-1}=\operatorname{Socle}(G)$. Also $k+k x^{-1} \subset G$ is an essential extension (where we recall that $x^{2} \cdot x^{-3}=0$ ). Hence $G$ is an essential colifting of the $\frac{R}{x^{2} R}$-module $k+k x^{-1}$. We also note that $E(G) \cong E(k) \oplus E(k)$. So $G$ is not an injective $R$-module.

We now recall from Northcott [12] that $E_{R}(k)=k+k x^{-2}+k x^{-3}+\cdots$ where $x^{2} \cdot x^{-3}=0$. So the imbedding $G \subset E(G)$ is given by

$$
G \stackrel{(1, x)}{\rightarrow} E(k) \oplus E(k)
$$

where $1: G \subset E(k)$ maps $x^{-1}$ to 0 but is an identity on $1, x^{-2}, x^{-3}, \cdots$ and $x \cdot x^{-2}=0$.

We now consider $k x^{-1} \subset G$. We get the imbedding $\underset{k x^{-1}}{G} \subset E(k) \oplus \frac{E(k)}{k}$. But $\underset{k x-1}{\frac{G}{k}} \cong E(k)$. So we have that the sequence $0 \rightarrow \frac{G}{k x^{-1}} \rightarrow E(k) \oplus \frac{E(k)}{k} \rightarrow \frac{E(k) \oplus E(k)}{G} \rightarrow 0$ is split exact. But $k x^{-1} \subset \operatorname{Hom}_{R}\left(\frac{R}{x^{2} R}, G\right) \subset G$. So the sequence

$$
0 \rightarrow \frac{G}{\operatorname{Hom}_{R}\left(\frac{R}{x^{2} R}, G\right)} \rightarrow \frac{E(k) \oplus E(k)}{\operatorname{Hom}_{R}\left(\frac{R}{x^{2} R}, G\right)} \rightarrow \frac{E(k) \oplus E(k)}{G} \rightarrow 0
$$

is split exact. Therefore,

$$
0 \rightarrow G \rightarrow \frac{E(k) \oplus E(k)}{\operatorname{Hom}_{R}\left(\frac{R}{x^{2} R}, G\right)} \rightarrow \frac{E(k) \oplus E(k)}{G} \rightarrow 0
$$

is also split exact. But then we have the following commutative diagram 


$$
\begin{aligned}
& \begin{array}{lllll} 
& 0 & & 0 & \\
& \downarrow & & \downarrow & \\
& \downarrow & x^{2} & & \\
& G & \rightarrow & G & \rightarrow 0
\end{array}
\end{aligned}
$$

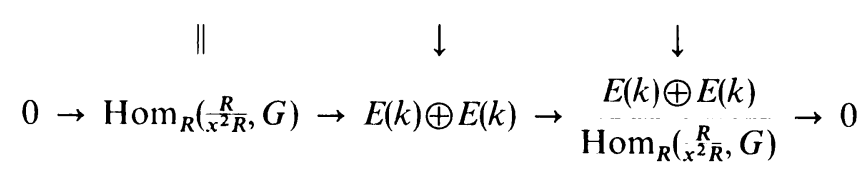

$$
\begin{aligned}
& \begin{array}{cc}
\downarrow & \downarrow \\
\frac{E(k) \oplus E(k)}{G}= & \frac{E(k) \oplus E(k)}{G}
\end{array} \\
& \downarrow
\end{aligned}
$$

with the last vertical sequence split exact. Hence $x^{2} \cdot \operatorname{Ext}^{1}(, G)=0$ and so $r_{x^{2}}(G)=1$. Thus

$$
G_{R}\left(\operatorname{Hom}_{R}\left(\frac{R}{x^{2} R}, G\right)\right) \cong G_{R}\left(k+k x^{-1}\right) \cong S_{R}\left(k+k x^{-1}\right) \cong G \oplus G
$$

by Lemma 4.1 and Theorem 4.2 since $S(G) \cong S^{-1}(G) \cong G$. Moreover, it follows from the above and Theorem 3.3 that every $R$-module $N$ with $r_{x}(N)=1$ and $\operatorname{Hom}_{R}\left(R / x^{2} R, N\right) \cong k+k x^{-1}$ is isomorphic to $G$.

We finally note that the $x^{\frac{R}{2}}-$-module $k$ is weakly coliftable to $R$. If $k$ were coliftable to $R$, then it is not hard to see that $k \cong \operatorname{Hom}(k, E(k))$ would be liftable to $R$. But $R=k\left[\left[x^{2}, x^{3}\right]\right]$ is not a discrete valuation ring. So $k$ is not liftable to $R$ (see Example 2 of Auslander-Ding-Soldberg[1, Proposition 3.2]), and thus $k$ is weakly coliftable but not coliftable to $R$.

We now study properties of essential coliftings. We start with the following.

Proposition 4.4. Let $L$ be an $R$-module. Then

1) Every essential colifting of $L$ is isomorphic to a submodule of $S_{R}(L)$.

2) If $M$ is an essential colifting of $L$, then $S_{R}(M)$ is an essential colifting of $S_{\bar{R}}(L)$.

Proof. If $M$ is an essential colifting of $L$ to $R$, then $E(M) \cong E(L)$ and so we have the following commutative diagram 


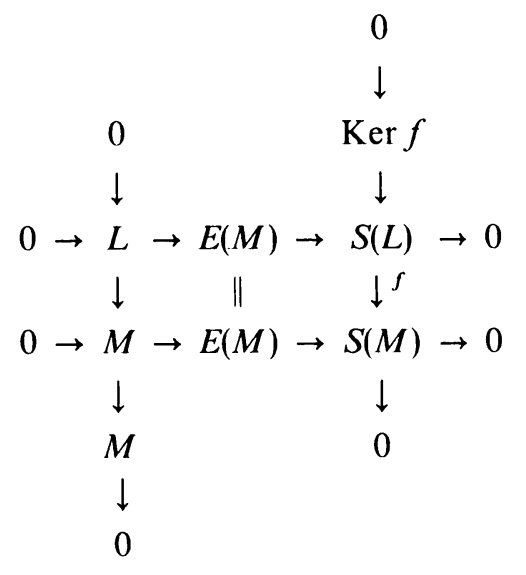

with $M \cong \operatorname{Ker} f$ and so (1) follows.

But $\operatorname{Ext}_{R}^{1}(\bar{R}, M)=0$ by assumption. So we have an exact sequence $0 \rightarrow \operatorname{Hom}_{R}(\bar{R}$, $M) \rightarrow \operatorname{Hom}_{R}(\bar{R}, E(M)) \rightarrow \operatorname{Hom}_{R}(\bar{R}, S(M)) \rightarrow 0$. Furthermore, $E_{\bar{R}}(L) \cong \operatorname{Hom}_{R}(\bar{R}, E(M))$. So $S_{\bar{R}}(L) \cong \operatorname{Hom}_{R}(\bar{R}, S(M))$. Moreover, $\operatorname{Ext}_{R}^{i}\left(\bar{R}, S_{R}(M)\right) \cong \operatorname{Ext}_{R}^{i+1}(\bar{R}, M)=0$ for all $i>0$ and so (2) follows.

Lemma 4.5. Let $R$ be $n$-Gorenstein and $M$ be a colifting of an $\bar{R}$-module. Then $p d_{\bar{R}} \operatorname{Hom}_{R}\left(\bar{R}, \frac{G(M)}{M}\right)<\infty$.

Proof. By Iwanaga [10], $i d_{R} \frac{G(M)}{M}<\infty$ since $p d_{R} \frac{G(M)}{M}<\infty$. So let $0 \rightarrow \frac{G(M)}{M} \rightarrow E^{0}$ $\rightarrow E^{1} \rightarrow \cdots \rightarrow E^{r} \rightarrow 0$ be an injective resolution of $\frac{G(M)}{M}$. Then the sequence $0 \rightarrow \operatorname{Hom}_{R}\left(\bar{R}, \frac{G(M)}{M}\right) \rightarrow \operatorname{Hom}_{R}\left(\bar{R}, E^{0}\right) \rightarrow \cdots \rightarrow \operatorname{Hom}_{R}\left(\bar{R}, E^{r}\right) \rightarrow 0$ is exact since $\operatorname{Ext}_{R}^{i}(\bar{R}$, $\left.\frac{G(M)}{M}\right) \cong \operatorname{Ext}_{R}^{i+1}(\bar{R}, M)=0$ for all $i \geq 1$. But for each $i, \operatorname{Hom}_{R}\left(\bar{R}, E^{i}\right)$ is an injective $\bar{R}$-module. So $i d_{\bar{R}} \operatorname{Hom}_{R}\left(\bar{R}, \frac{G(M)}{M}\right)<\infty$. But then the result follows since $\bar{R}$ is also Gorenstein.

Proposition 4.6. Let $R$ be $n$-Gorenstein and $M$ be an essential colifting of an $\bar{R}$-module. Then

$$
\operatorname{Hom}_{R}\left(\bar{R}, G_{R}(M)\right) \cong G_{\bar{R}}\left(\operatorname{Hom}_{R}(\bar{R}, M)\right)
$$

Proof. $\operatorname{Hom}_{R}(\bar{R}, M)$ is an essential $R$-submodule of $M$ by assumption and $M$ is a Gorenstein essential submodule of $G(M)$ by Enochs-Jenda [7, Theorem 3.3]. So it is easy to argue that $\operatorname{Hom}_{R}(\bar{R}, M)$ is a Gorenstein essential submodule of $G(M)$. But $\operatorname{Hom}_{R}(\bar{R}, M) \subseteq \operatorname{Hom}_{R}(\bar{R}, G(M)) \subseteq G(M)$. So $\operatorname{Hom}_{R}(\bar{R}, M) \subseteq \operatorname{Hom}_{R}(\bar{R}, G(M))$ is a Gorestein essential extension as $R$-modules and hence as $\bar{R}$-modules.

Since $M$ is a colifting, we have the exact sequence

$$
0 \rightarrow \operatorname{Hom}_{R}(\bar{R}, M) \rightarrow \operatorname{Hom}_{R}(\bar{R}, G(M)) \rightarrow \operatorname{Hom}_{R}\left(\bar{R}, \frac{G(M)}{M}\right) \rightarrow 0 .
$$


But $p d_{R} \operatorname{Hom}_{R}\left(\bar{R}, \frac{G(M)}{M}\right)<\infty$ by Lemma 4.5 above and $\operatorname{Hom}_{R}(\bar{R}, G(M))$ is a Gorenstein injective $\bar{R}$-module by Enochs-Jenda [6, Lemma 3.1]. Hence $\operatorname{Hom}_{R}(\bar{R}, G(M))$ is a Gorenstein essential Gorenstein injective extension of the $\bar{R}$-module $\operatorname{Hom}_{R}(\bar{R}, G(M))$ and so is the Gorenstein injective envelope by Theorem 3.3 of [7].

The following result is dual to Proposition 5.2 of Auslander-Ding-Soldberg [1].

Theorem 4.7. Let $R$ be $n$-Gorenstein and $L$ be an $\bar{R}$-module. If $L$ is essentially coliftable to $R$, then $G_{\bar{R}}(L)$ and $G_{\bar{R}}(L) / L$ are coliftable to $R$.

Proof. If $M$ is an essential colifting of $L$ to $R$, then $G_{\bar{R}}(L) \cong \operatorname{Hom}_{R}(\bar{R}, G(M))$ by Proposition 4.6 above and so $G_{\bar{R}}(L) / L \cong \operatorname{Hom}_{R}\left(\bar{R}, \frac{G(M)}{M}\right)$. But $\operatorname{Ext}_{R}^{i}\left(\bar{R}, \frac{G(M)}{M}\right)$ $\cong \operatorname{Ext}_{R}^{i+1}(\bar{R}, M)=0$ for all $i>0$. Thus $G(M)$ and $\frac{G(M)}{M}$ are coliftings of $G_{\bar{R}}(L)$ and $\frac{G_{R}(L)}{L}$ to $R$, respectively.

As a consequence, we get the following Gorenstein version of part 2 of Proposition 4.4 above.

Corollary 4.8. Let $R$ be $n$-Gorenstein and $L$ be an $\bar{R}$-module. If $L$ is coliftable to an $R$-module whose Gorenstein injective envelope is an essential extension of $L$, then $G_{\bar{R}}(L)$ and ${ }^{G_{R}(L)}$ are essentially coliftable to $R$.

To prove the converse of this corollary, we need the following easy

Lemma 4.9. Let $L$ be an $\bar{R}$-module. Then $L$ is an injective $\bar{R}$-module that is essentially coliftable to a Gorenstein injective $R$-module if and only if $L$ $\cong \operatorname{Hom}_{R}\left(\bar{R}, E_{R}(L)\right)$.

Proof. If $L$ is coliftable to $R$, then $L \cong \operatorname{Hom}_{R}(\bar{R}, G)$ for some Gorenstein injective $R$-module $G$. But $i d_{R} L=1$ since $L$ is an injective $\bar{R}$-module. So $\operatorname{Ext}_{R}^{1}\left(\frac{E(L)}{L}, G\right)=0$ and thus $E(L)$ is a summand of $G$. But then $G \cong E(L)$ since $L \subset G$ is essential.

Conversely, $L$ is an injective $\bar{R}$-module and $E(L)$ is an essential colifting of $L$.

Theorem 4.10. Let $R$ be 2-Gorenstein and $L$ be an $\bar{R}$-module. Then $L$ is coliftable to an $R$-module whose Gorenstein injective envelope is an essential extension of $L$ if and only if $G_{\bar{R}}(L)$ is coliftable to a Gorenstein injective $R$-module that is an essential extension of $L, G_{\bar{R}}(L) / L$ is essentially coliftable to $R$, and the image of the lifting of the natural map $G_{\bar{R}}(L) \rightarrow \frac{G_{R}(L)}{L}$ to $R$ has finite projective dimension.

Proof. The only if part is essentially Corollary 4.8 above.

We now prove the if part. If $i d R=1$, then $i d_{\bar{R}} \bar{R}=0$ and so $G_{\bar{R}}(L)=L$. Thus $L$ is coliftable to a Gorenstein injective $R$-module that is an essential extension of 
$L$ by assumption. If $i d R=2$, then $i d_{\bar{R}} \bar{R} \leq 1$ and so $C=\frac{G_{R}(L)}{L}$ is an injective $\bar{R}$-module by Enochs-Jenda [5, Theorem 3.3]. Hence the injective envelope of $C$ is its colifting by the Lemma above. Now let $G$ be a Gorenstein injective colifting of $G_{\bar{R}}(L)$ to $R$ with $L \subseteq G$ essential. Then we have the following commutative diagram

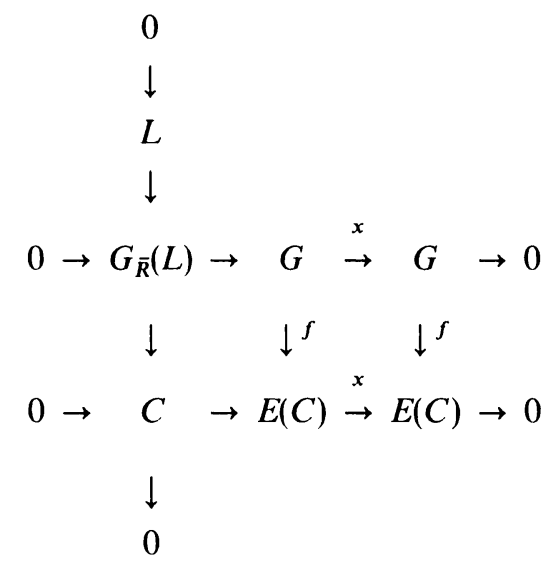

So we have an exact sequence $0 \rightarrow L \rightarrow \operatorname{Ker} f \stackrel{x}{\rightarrow} \operatorname{Ker} f \rightarrow 0$. Thus $L \cong \operatorname{Hom}_{R}(\bar{R}, \operatorname{Ker} f)$ and $\operatorname{Ext}^{1}(\bar{R}, \operatorname{Ker} f)=0$. Moreover, $\operatorname{Ext}_{R}^{i}(\bar{R}, \operatorname{Ker} f)=0$ for all $i \geq 2$ since $p d \bar{R} \leq 1$. So Ker $f$ is a colifting of $L$. But $p d f(G)<\infty$ by assumption. So $G \cong G(\operatorname{Ker} f) \oplus E$ for some injective $R$-module $E$. But then $E=0$ since $L \subseteq G$ is essential. So we are done.

We now conclude the section by characterizing $\bar{R}$-modules that are weakly coliftable to Gorenstein injective $R$-modules.

Theorem 4.11. The following are equivalent for an $\bar{R}$-module $L$.

1) $L$ is weakly coliftable to a Gorenstein injective $R$-module.

2) $L$ is a direct summand of $\operatorname{Hom}_{R}\left(\bar{R}, G_{R}(L)\right)$.

Moreover, if $R$ is 1-Gorenstein, then each of the above statements is equivalent to

3) $\operatorname{Hom}_{R}\left(\bar{R}, S_{R}(L)\right) \cong L \oplus S_{\bar{R}}(L)$.

Proof. $\quad 1 \Rightarrow 2$. Let $M$ be a Gorenstein injective $R$-module that is a weak colifting of $L$. Then we have the following commutative diagram

$$
\begin{aligned}
& 0 \rightarrow L \rightarrow G_{R}(L) \rightarrow \frac{G_{R}(L)}{L} \rightarrow 0 \\
& \begin{array}{l}
\| \\
0 \rightarrow L \rightarrow M \\
\end{array} \rightarrow \frac{\downarrow}{L} \rightarrow 0
\end{aligned}
$$

We now apply $\operatorname{Hom}_{R}(\bar{R},-)$ to the diagram to get the following commutative diagram since $\operatorname{Hom}_{R}(\bar{R}, L) \cong \operatorname{Ext}_{R}^{1}(\bar{R}, L) \cong L$. 


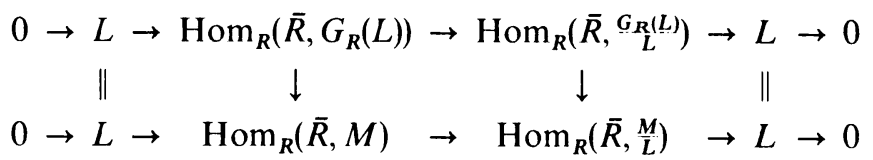

But $L$ is a direct summand of $\operatorname{Hom}(\bar{R}, M)$. So $L$ is a direct summand of $\operatorname{Hom}_{R}\left(\bar{R}, G_{R}(L)\right)$.

$2 \Rightarrow 1$ follows from the definition.

$1 \Rightarrow 3$ follows from Lemma 3.2.

$3 \Rightarrow 1$ is trivial since $S_{R}(L)$ is a Gorenstein injective R-module because $R$ is 1 -Gorenstein.

\author{
DePARTMENT OF MATHEMATICS \\ UNIVERSITY OF KENTUCKY \\ LEXINGTON \\ KY 40506-0027 \\ USA
}

Department of Discrete and Statistical Sciences 120 Mathematics ANNEX

AUBURN UNIVERSITY

AL 36849-5307

USA

\title{
References
}

[1] A. Auslander, S. Ding and O. Soldberg, Liftings and weak liftings of modules, J. Algebra, 156 (1993), 273-317.

[2] S. Ding and O. Soldberg, Maranda theorem and liftings of modules, Comm. Algebra, 21 (1993), 1161-1187.

[3] E. Enochs, Injective and flat covers, envelopes and resolvents, Israel J. Math, 39 (1981), 33-38.

[4] E. Enochs and O. Jenda, Gorenstein injective and projective modules, Math. Z., 220 (1995), 611-633.

[5] E. Enochs and O. Jenda, Resolutions by Gorenstein injective and projective modules and modules of finite injective dimension over Gorenstein rings, Comm. Algebra, 23 (1995), 869-877.

[6] E. Enochs and O. Jenda, Gorenstein injective dimension and Tor-depth of modules, Archivier Mathematik, to appear.

[7] E. Enochs and O. Jenda, Gorenstein injective modules over Gorenstein rings, Comm. Algebra, to appear.

[ 8 ] E. Enochs, O. Jenda and J. Xu, Covers and envelopes over Gorenstein rings, Tsukuba J. Math., 20 (1996), 487-503.

[9] P. Hilton, Homotopy theory and duality, Gordon and Breach, New York, 1965.

[10] Y. Iwanaga, On rings with finite self-injective dimension II, Tsukuba J.Math., 4 (1980), 107-113.

[11] E. Kunz, The value-semigroup of a one-dimensional Gorenstein ring, Proc. Amer. Math. Soc., 25 (1970), 748-751.

[12] D. Northcott, Injective envelopes and inverse polynomials, J. London Math. Soc., 8(1974), 290-296. 